\title{
44388 - OSA QUESTIONNAIRE: A NEW TOOL FOR SCREENING OBSTRUCTIVE SLEEP APNEA
}

\author{
Frances Chung, University of Toronto, University Health Network, Toronto, ON, Canada; \\ Balaji Yegneswaran, University of Toronto, University Health Network; \\ Sharon Chung, University of Toronto, University Health Network; \\ S Islam, University of Toronto, University Health Network; \\ A Khajehdehi, University of Toronto, University Health Network; \\ C Shapiro, University of Toronto, University Health Network;
}

Introduction: Obstructive sleep apnea (OSA) affects millions of middle-aged men and women. At least $80 \%$ of moderate to severe OSA patients are undiagnosed before surgery and thus remain untreated perioperatively(1). In the preadmission clinics, a concise screening tool for OSA will be a great help for anesthesiologists. Berlin questionnaire (BQ) was developed for the screening of OSA in a primary care setting. We developed the OSA questionnaire (OSAQ) a new concise screening tool for OSA. The objective of this study was to validate the BQ and OSA questionnaire for the screening of OSA in surgical patients by comparing these questionnaires to polysomnographic (PSG) study, the gold standard in diagnosing OSA.

Methods: Hospital ethics approval and informed consent was obtained. All eligible preadmission clinic patients, age\&\#8805;18 years, ASA I-IV, were screened for OSA using BQ and OSA questionnaire. BQ consists of 9 item regarding snoring behavior, wake-time sleepiness or fatigue and the presence of obesity or hypertension. OSA questionnaire contains 4 questions: "Do you snore loudly (louder than talking or loud enough to be heard through closed doors)?", "Do you often feel tired, fatigued or sleepy during daytime?", "Do you have or are you being treated for high blood pressure?" and "Has anyone noticed you stop breathing during your sleep?" Based on response to questions, patients were classified as high risk if they responded yes to two or more questions to OSAQ. All patients screened were invited for PSG. PSG scorers were blinded to the results of the 2 questionnaires. McNemar's test was used to analyse the agreement between the 2 methods and PSG data.

Results: 2426 patients were screened with BQ and OSA questionnaire. BQ identified $23.4 \%$ (567/2426) whereas OSA questionnaire identified 22.3\% (541/2426) as being at high risk for OSA. 2003 patients were invited for PSG, 223 patients gave informed consent and 1780 patients refused. 94 patients completed PSG. Mean age $57 \pm 13$ years, M:F - 56\%:44\% and ASA I:7.4\%, II:50\%, III:40.4\%, IV:2.2\%.With PSG, 39\% (55/141) of patients were diagnosed to have moderate-severe OSA, AHI $>15 / \mathrm{hr}$ (table). Sensitivity, specificity, positive predictive value, negative predictive value of BQ was $0.69,0.45,0.47$ and 0.68 and of OSA questionnaire 0.71, $0.49,0.50$ and 0.71 . Using McNemar's test, both questionnaires agreed with AHI in diagnosing OSA patients $(\mathrm{p}<0.05)$.

Discussion: OSA questionnaire has better sensitivity and specificity than Berlin Questionnaire and both have been validated to screen surgical patient for OSA.

Reference: 
1 Ann Intern Med. 1999; 131: 485-91.

Table - Questionnaires and PSG results

\begin{tabular}{|c|c|c|c|c|}
\hline $\mathrm{n}=94$ & \multicolumn{2}{|c|}{ BQ } & \multicolumn{2}{c|}{ OSA Questionnaire } \\
\hline PSG & $\begin{array}{c}\text { High Risk } \\
(\mathrm{n}=57)\end{array}$ & $\begin{array}{c}\text { Low Risk } \\
(\mathrm{n}=37)\end{array}$ & $\begin{array}{c}\text { High Risk } \\
(\mathrm{n}=56)\end{array}$ & $\begin{array}{c}\text { Low Risk } \\
(\mathrm{n}=38)\end{array}$ \\
\hline $\begin{array}{c}\text { AHD }>15 / \mathrm{hr}(\mathrm{n}=39) \\
(\text { Moderate OSA) }\end{array}$ & 27 & 12 & 28 & 11 \\
\hline AHI $\leq 15 / \mathrm{hr}(\mathrm{n}=55)$ & 30 & 25 & 28 & 27 \\
\hline
\end{tabular}

$\mathrm{n}$ - number of patients 